\title{
Design of Control System for Intelligent Self-Service Cabinet
}

\author{
Xiangfei Meng ${ }^{1,}$, Xin $\mathrm{Li}^{1, \mathrm{~b}}$, Qiming $\mathrm{Gu}^{1, \mathrm{c}}$ \\ ${ }^{1}$ Changshu Institute of Technology, School of Electrical and Automation Engineering, Changshu \\ 215500, China \\ aemail: mxf0316@163.com, bemail: 93238016@qq.com \\ cemail:2507563733@qq.com
}

Keywords: Intelligent Self-Service Cabinet; Fresh distribution; Controller; RS-485 Bus

\begin{abstract}
The Intelligent Self-Service Cabinet is an important device which developed for convenient delivery of fresh distribution fields. In order to meet the needs of the users, this paper is based on Cortex-M3 to cater the design requirements of the Intelligent Self-Service Cabinet. The Host-Controller communicates with the Sub-Controller by RS-485 Bus. Once the Sub-Controller received the instructions from the Host-Controller, it can set the communication address and display data; in addition, it can also open and close all the cells, or detect temperature and adjust the temperature; what is more, it can use the infrared sensor to detect items and so on. All of those are by means of the peripheral module circuit. When we put them together, thereby we can use the Sub-Controller to control the Intelligent Self-Service Cabinet.
\end{abstract}

\section{Introduction}

Especially the last mile of the distribution about fresh product has been difficult to break through the technical bottleneck, which gives rise to the sale of fresh electricity supplier has brought a great deal of trouble. In view of the above shortcomings, smart fresh from mentioning cabinet system provides a cold storage type self lockers in machinery from mentioning cabinet is composed of several independent and a box door closed container, with refrigeration equipment and control system of intelligent cabinets from mentioning fresh complete from mentioning cabinet system, can from the cold chain storage on the locomotive after fresh products, and can provide consumers with fresh products 24 hours pick up service, avoid the attendant direct distribution to consumers in the process, so as to solve the logistics distribution chain of fresh products distribution problem, meet the development needs of the new logistics system with low energy consumption.

\section{Design of Control System}

In order to meet the needs of the customers, the intelligent Self-Service cabinet should be able to communicate with the master and slave logic based on the realization of the function of the main controller and the controller. In order to facilitate the function of intelligent self - extracting cabinet, flexible configuration and routing, installation and maintenance, the design of the system adopts master-slave bus system architecture. The overall design block diagram of the system is shown in figure 1.

A set of intelligent Self-Service cabinet is provided with 10 sub cabinets, and is controlled by a sub controller, and the main controller can be connected with a plurality of sub controllers through the field bus, so as to realize the convenient module expansion function.

The main controller by combining storage module, voice module, card module, barcode scanning module, wireless communication module, keyboard module, LCD module main control circuit, and then realize the local storage, voice broadcast, IC card identification, barcode scanning, wireless communication, human-computer interaction and other functions.

The utility model is provided with a 10 way cabinet control port, and the corresponding $10 \mathrm{sub}$ cabinets are respectively controlled. Each sub controller for detecting and controlling temperature and humidity of each cell, infrared object detection and gated lock gate, data transmit through Fieldbus with the main controller, without sensor signal line is connected to the main controller, 
greatly reducing the number of line and line length.

Each sub controller is only connected with the main controller through two lines, and the main controller of the data exchange, a main controller can mount the 255 cells, fully meet the application requirements, and walk the line less, cost-effective.

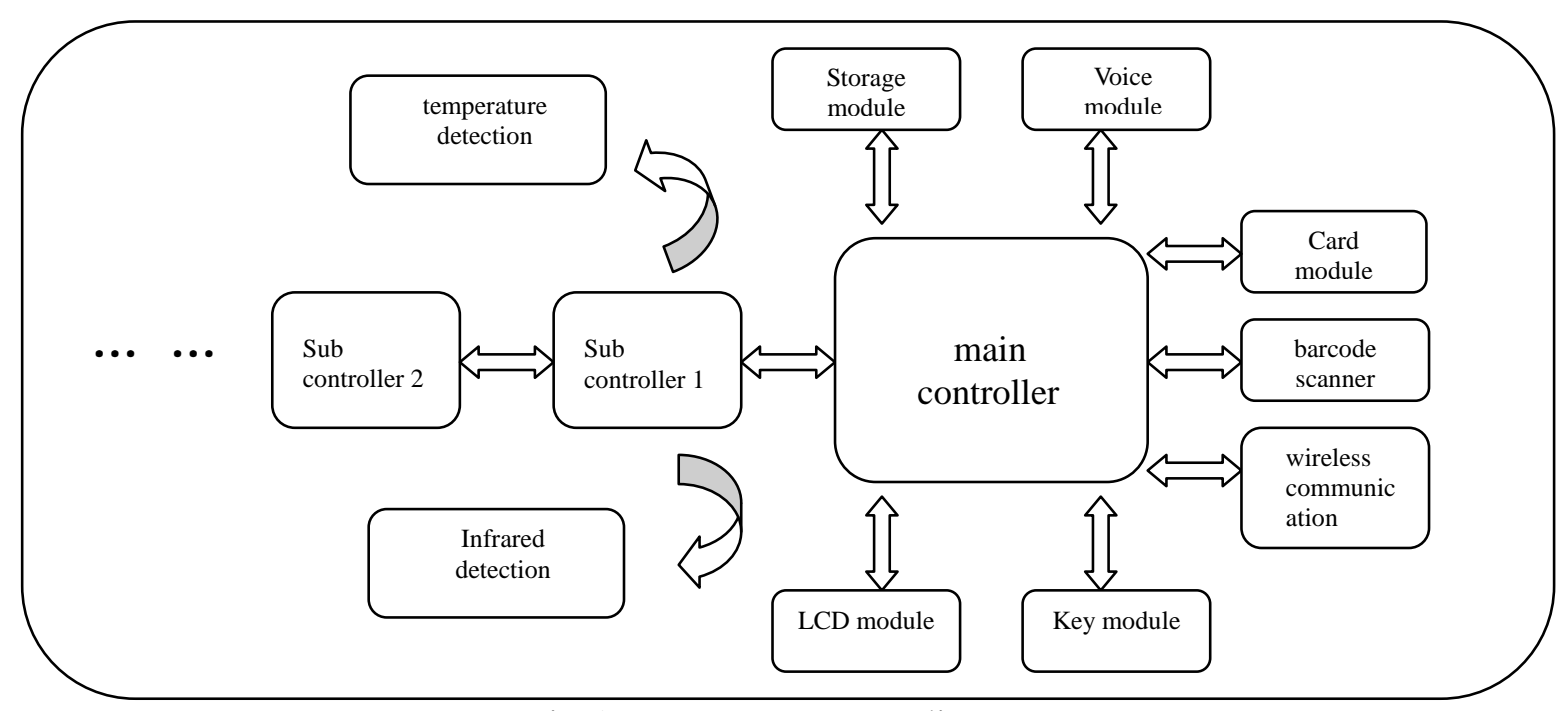

Fig.1. system structure diagram

\section{Hardware circuit design}

\section{Design of main power supply.}

After power up, $220 \mathrm{~V}$ city electricity through the bridge rectifier filter, about $300 \mathrm{~V}$ of the DC voltage in the capacitor $\mathrm{C} 1$ both ends appear, through the high frequency transformer $\mathrm{T}$ of the original side of the N1 plus WS157 in the D, so that the internal bias circuit work. The anti peak clamping circuit is composed of a resistor R1, a capacitor C2 and a diode VD5, which can improve the conversion efficiency by reducing the reverse peak voltage of the $\mathrm{D}$ terminal. $\mathrm{T}$ two side winding N2 output square wave voltage through VD7, C4, C5, Lo high frequency rectifier output DC voltage for the use of $12 \mathrm{~V} / 0.5 \mathrm{~A}$. The sampling voltage is composed of the voltage of the feedback winding and the $\mathrm{C}$ end of the WS157, which is filtered by a diode VD6 and a capacitor C3. When the input voltage is decreased or increased load caused by the output voltage drop, and drop the primary side feedback voltage winding N3, injected current sampling C end is therefore reduced, compared by internal pulse width modulation circuit, a linear increase of power switch duty cycle, so that the output DC voltage remained relatively average the same. Among them, the resistance R2, the capacitance C3 and the voltage of the primary side winding N3 determine the control state of the control loop, and the duty cycle is 0.47 when the load is full[1]. The switching power supply type voltage stabilizing circuit is shown in figure 2 .
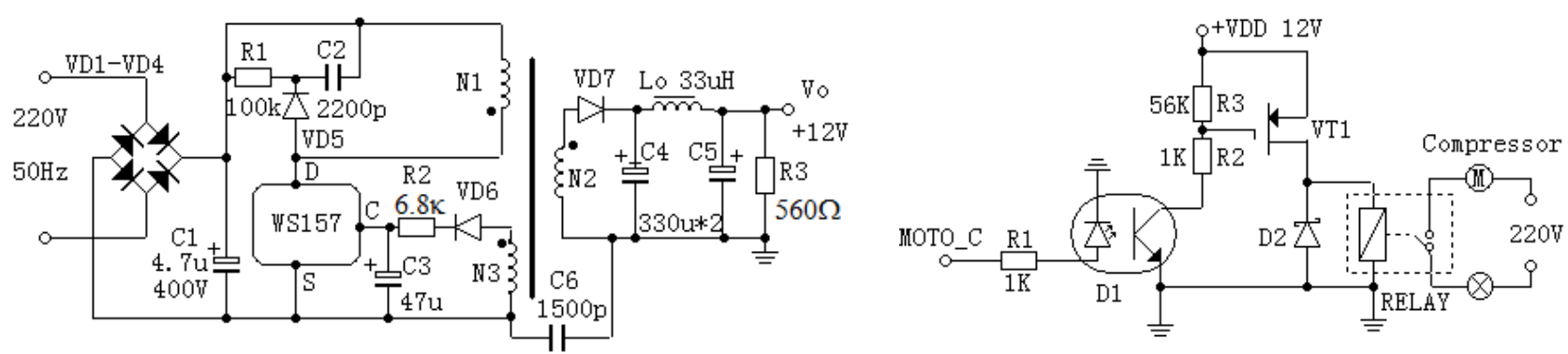

Fig.2. Switching power supply type voltage stabilizing circuit Fig.3. Compressor control circuit

Three terminal integrated switching power supply WS157 includes the reference voltage source, error voltage amplification, PWM comparison, constant current source driver circuit, built-in $100 \mathrm{kHz}$ oscillator, with over temperature, over-current protection function. This configuration also 
allows it to have a high degree of integration, simple peripheral circuits, improve the protection function of the AC input voltage range. Using $100 \mathrm{kHz}$ switching frequency, low loss, high power efficiency, strong anti-interference ability, flexible use, and so on, so it becomes the first choice of the power supply circuit.

\section{Design of temperature control circuit.}

Using DHT11 digital temperature and humidity sensors as sensor for detecting the temperature in the cabinet, the temperature and humidity sensor using digital modules acquisition dedicated, and has been carried out in the digital calibration output signal, with fast response, strong anti-interference ability and high cost performance, its high reliability and long-term stability to meet the requirements of our design[2].

In order to achieve the purpose of controlling temperature, the temperature and humidity sensor temperature and humidity information in the cabinet to the sub controller, controller software after data processing, get the exact value, compared with the expected value, control of temperature and humidity to determine what measures should be taken. The compressor control circuit is shown in figure 3.

\section{Design of human computer interaction circuit.}

The design of human-computer interaction greatly facilitates the debugging work of designers. As the sub controller interface is limited, so the host control two machine from the strategy, from the machine in connection with the corresponding unit cabinet, by switch control selection from the corresponding machine is connected to the host, use the same bus control output is different, in order to achieve the purpose of controlling the needle[3[4]]. The digital tube display circuit and dialing circuit is shown in figure 4 and 5.

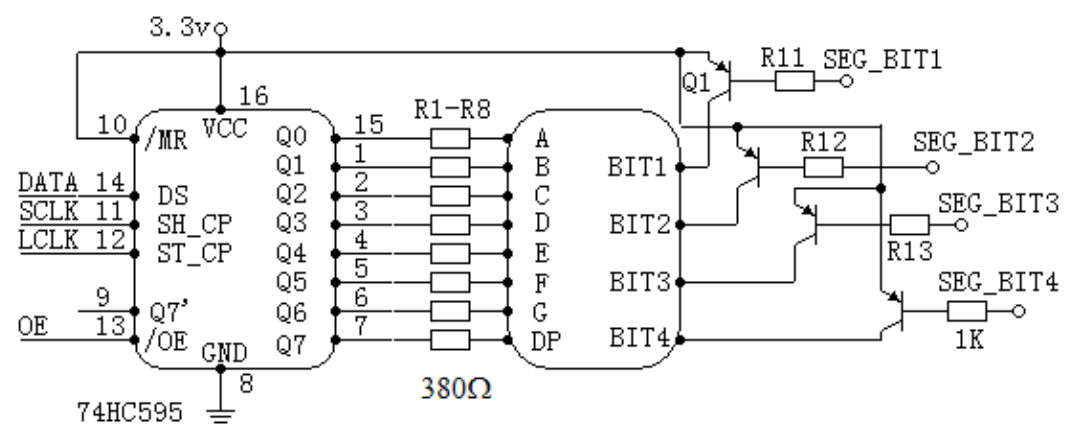

Fig.4. Digital tube display circuit
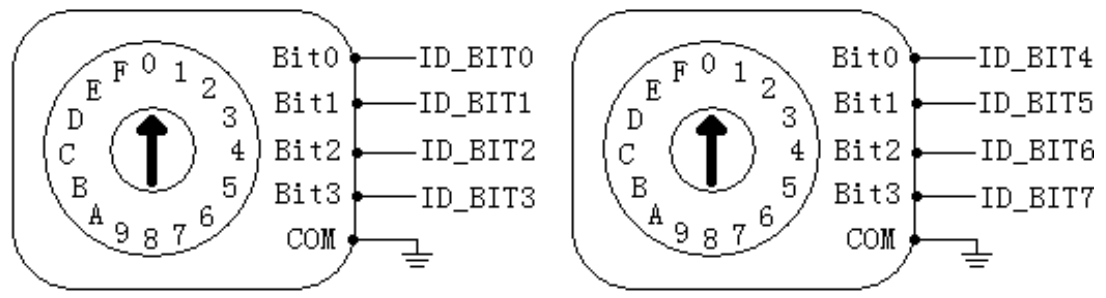

Fig.5. Dialing circuit

\section{Design of synchronous communication circuit.}

In order to realize the synergy between the host and slave communication, you can use the RS-232 interface to connect the host and slave, but because the standard RS-232 interface introduced earlier, its transmission rate is slow, the disadvantage of short transmission distance can not meet the requirements of multi computer communication system in industrial applications in many cases, the RS-485 interface standard in practical application often using the data of strong anti-interference ability, wide range of communication and software design without the modification[5] [6]. The RS485 communication circuit is shown in figure 6. 


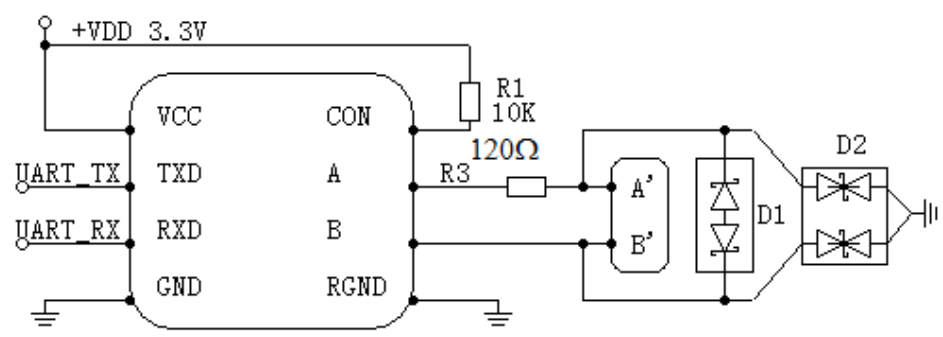

Fig.6. RS485 communication circuit

\section{Stability test}

The main controller and sub controller complete from mentioning cabinet system, the stability test for a long time, the time span has been more than 2 months, found a number of details wrong during one repair success, in addition, there is a fatal defect of low probability, this phenomenon is: defects in operation. All the operation failure, power failure to restart to recover, even more serious is that there are several problems after the restart, all the password is invalid, can not enter the interface, through the monitoring data in the MCU program, the data stored in the FLASH chip is destroyed.

After repeated inspection and debugging procedures, no fatal error, initially suspected to be connected with the operating system scheduler, the scheduler function error, lead to disorderly execution and MCU affect the normal operation of the system or even destroy data. Access to relevant information, to further doubt is the task allocation of stack space is not enough, the stack space detection function through the operating system with the monitoring of each task stack space usage and found slightly human-computer interactive task stack space is insufficient, the task stack space to continue testing, normal operation, is not there a fatal error.

\section{Conclusion}

In the future, with the rapid development of science and technology industry convenient, people rely on intelligent products facilities will grow with each passing day intelligent industrial system, instead of the conventional mechanical labor, then, intelligence from mentioning cabinet development will rapidly forward, and more convenient and more intelligent from mentioning cabinet will be pregnant born.

\section{Acknowledgement}

In this paper, the research was sponsored by the Jiangsu province industry university research joint innovation fund (Project No.BY2015043-01).

\section{References}

[1] Kang Huaguang. Electronic technology foundation [M]. fifth ed. higher education press, 2006.1.

[2] Dohler M. cooperative communication: physical layer, channel model and system implementation [M]. Machinery Industry Press, 2011.3.

[3] LFloyd T. Digital Fundamentals[M]. Tenth Edition. Publishing House of electronics industry, 2014.1.

[4] Chen Qijun. Embedded system and its application [M]. Shanghai: Tongji University press.2014.

[5] Feng Ziling.RS485 bus communication protocol design and implementation [J]. Computer Engineering, 2012, 56(10) 50-53.

[6] Metrologic instruments,Inc.MetroSelect Single-Line Configuration Guide.2007. 